\title{
Space Charge Correction on Emittance Measurement of Low Energy Electron Beams
}

\author{
Colleen J. Treado \\ Office of Science, Science Undergraduate Laboratory Internship (SULI) \\ University of Massachusetts Amherst \\ SLAC National Accelerator Laboratory \\ Stanford, CA 94025
}

August 12, 2011

Prepared in partial fulfillment of the requirements of the Office of Science, Department of Energy's Science Undergraduate Laboratory Internship under the direction of Cecile LimborgDeprey at the Accelerator Division, SLAC National Accelerator Laboratory.

Participant:

Signature

Research Advisor:

Signature 


\section{TABLE OF CONTENTS}

Abstract

$\begin{array}{ll}\text { Introduction } & 1\end{array}$

Materials and Methods $\quad 5$

$\begin{array}{ll}\text { Results } & 11\end{array}$

$\begin{array}{ll}\text { Discussion and Conclusions } & 13\end{array}$

$\begin{array}{ll}\text { Acknowledgements } & 15\end{array}$

$\begin{array}{ll}\text { References } & 15\end{array}$ 


\begin{abstract}
Space Charge Correction on Emittance Measurement of Low Energy Electron Beams. COLLEEN J. TREADO (University of Massachusetts Amherst, Amherst, MA 01003) CECILE LIMBORGDEPREY (Accelerator Division, SLAC National Accelerator Laboratory, Stanford, CA 94025)
\end{abstract}

The goal of any particle accelerator is to optimize the transport of a charged particle beam along a set path by confining the beam to a small region close to the design trajectory and directing it accurately along the beamline. To do so in the simplest fashion, accelerators use a system of magnets that exert approximately linear electromagnetic forces on the charged beam. These electromagnets bend the beam along the desired path, in the case of bending magnets, and constrain the beam to the desired area through alternating focusing and defocusing effects, in the case of quadrupole magnets. We can model the transport of such a beam through transfer matrices representing the actions of the various beamline elements. However, space charge effects, produced from self electric fields within the beam, defocus the beam and must be accounted for in the calculation of beam emittance. We present below the preliminary results of a MATLAB code built to model the transport of a charged particle beam through an accelerator and measure the emittance under the influence of space charge effects. We demonstrate the method of correctly calculating the emittance of a beam under space charge effects using a least square fit to determine the initial properties of the beam given the beam size measured at a specific point after transport. 


\section{INTRODUCTION}

Accelerator physics studies the optimization of the acceleration and transport of charged particle beams. We define a beam through its distribution in phase space using the particles spatial coordinates and conjugate coordinates, or normalized momenta. The spatial coordinates, $(x, y, z)$, correspond to the displacements of the particles from a synchronous particle, corresponding to the centroid of the beam and having a specified design velocity and trajectory. The z-coordinate, aligned with the design trajectory, represents the longitudinal position of the beam along the accelerator, thus leaving the xy-plane to represent the transverse plane. The normalized momenta, $\left(x^{\prime}, y^{\prime}, z^{\prime}\right)$, are taken with respect to the longitudinal position of the synchronous particle, $s$, rather than time [1].

In a particle accelerator, the beam is guided along a desired path by electromagnetic forces produced by components of the accelerator. These forces, known as Lorentz forces and defined as

$$
\mathbf{F}=q \mathbf{E}+\frac{q}{c}(\mathbf{v} \times \mathbf{B})
$$

where $q$ is the charge of a single particle, $\mathbf{E}$ is the electric field, $\mathbf{v}$ is the velocity of the synchronous particle, and $\mathbf{B}$ is the magnetic field, also confine the beam to a narrow region. To good approximation, all of the fields contributing to the Lorentz forces can be assumed as linear, and the evolution of the trajectory of the charged particle beam due to such linear Lorentz forces is known as linear beam dynamics. Such forces are produced by beam transport magnets, specifically quadrupoles and bending magnets, the two of which alone completely achieve the basic principles of linear beam dynamics, enabling beam transport systems to consist solely of linear components [2]. Thus, we can approximate the equations of motion governing the transport of the charged particle beam through the accelerator to 
first order as harmonic oscillators, as follows:

$$
\begin{aligned}
x^{\prime \prime}+\frac{\kappa_{x}}{\gamma m c^{2} \beta^{2}} x & =0, \\
y^{\prime \prime}+\frac{\kappa_{y}}{\gamma m c^{2} \beta^{2}} y & =0, \\
z^{\prime \prime}+\frac{\kappa_{z}}{\gamma m c^{2} \beta^{2}} z & =0,
\end{aligned}
$$

where $\beta=\frac{v}{c}$, with $c$ being the speed of light, $\gamma=\frac{1}{\sqrt{1-\beta^{2}}}, m$ is the mass of a particle, $\kappa_{\alpha}$ represents the action of the transport element on the beam, and primes represent differentiation with respect to $s$ [1].

An important concept in accelerator physics is that of the rms envelope. The rms envelopes represent the size of the beam and are described by

$$
\begin{aligned}
& \tilde{x}=\sqrt{<x^{2}>}, \\
& \tilde{y}=\sqrt{<y^{2}>}, \\
& \tilde{z}=\sqrt{<z^{2}>},
\end{aligned}
$$

for a centered beam [1]. More important yet is the beam emittance, which represents the volume in phase space occupied by the rms particle beam envelopes and is defined as

$$
\epsilon_{x}=\sqrt{<x^{2}><x^{\prime 2}>-<x x^{\prime}>^{2}}
$$

with similar definitions in the $y$ and $z$ planes. Under the influence of only linear forces, the emittance is unchanged by the motion of the beam, and a changing emittance indicates undesired nonlinear forces acting on the beam. Therefore, producing and maintaining a small transverse beam emittance is among the fundamental objectives in the design and 
operation of a high quality accelerator.

While it is possible to describe the transformation of the particle beam through transportation of individual particles, this method is often cumbersome and time-consuming. We demonstrate that it is possible and effective to instead directly transport only the second order moments of the beam. We surround the two-dimensional projection of the particle beam distribution with an ellipse, defined by:

$$
\gamma_{x} x^{2}+2 \alpha_{x} x x^{\prime}+\beta_{x} x^{2}=\epsilon_{x}
$$

for the $\mathrm{x}$ projection, where $\alpha_{x}, \beta_{x}$, and $\gamma_{x}$ are the Courant-Snyder, or Twiss, parameters, defined below:

$$
\begin{aligned}
\alpha_{x} & =\frac{<x x^{\prime}>}{\epsilon_{x}}, \\
\beta_{x} & =\frac{<x^{2}>}{\epsilon_{x}}, \\
\gamma_{x} & =\frac{<x^{\prime 2}>}{\epsilon_{x}},
\end{aligned}
$$

[1]. The ellipse has an area of $A=\pi \epsilon_{x}$, where $\epsilon_{x}$ is the emittance in x [2]. Similar ellipses and parameters exist in the $y$ and $z$ planes. Liouville's Theorem, which states that the density of particles remains constant along the beam transport, or

$$
\frac{d}{d s} f\left(x(s), x^{\prime}(s), y(s), y^{\prime}(s), z(s), z^{\prime}(s)\right)=0
$$

where $f$ is the density function of the phase space coordinates [1], ensures that the particles contained within the ellipse remain within the ellipse and that the ellipse's area, and thus the emittance of the beam, remains constant throughout the entire transport along the beam. Therefore, it is possible to describe the transformation of the beam through the transformation of its Twiss parameters alone [2]. As the emittance remains unchanged, measuring the 
transformation of the Twiss parameters is equivalent to measuring the transformation of the beam's second order moments; therefore, we can model the transport of the charged particle beam through the transport of its second order moments.

A charged particle beam is subject to defocusing electrostatic forces between the charged particles and to focusing magnetic forces, due to the motion of the charged particles and dependent on the velocity of the beam. However, at low beam energies, the electric forces are greater than the magnetic forces; therefore, the self-fields generated from the electric forces non-negligibly defocus, and thus destabilize, the beam. The equations of motion are no longer homogeneous, instead described by

$$
\begin{gathered}
x^{\prime \prime}+\frac{\kappa_{x}}{\gamma m c^{2} \beta^{2}} x=K \frac{2 \pi \epsilon_{0}}{q N} E_{x}, \\
y^{\prime \prime}+\frac{\kappa_{y}}{\gamma m c^{2} \beta^{2}} y=K \frac{2 \pi \epsilon_{0}}{q N} E_{y}, \\
z^{\prime \prime}+\frac{\kappa_{z}}{\gamma m c^{2} \beta^{2}} z=\gamma^{2} K \frac{2 \pi \epsilon_{0}}{q N} E_{z},
\end{gathered}
$$

where $K=\frac{q N}{2 \pi \epsilon_{0}} \frac{1}{\gamma^{3} \beta^{2}} \frac{q}{m c^{2}}$ [1] is the beam perveance, $N$ is the total number of particles in the beam, and $\epsilon_{0}$ is the permittivity of free space. These space charge effects, represented by the inhomogenous term, must therefore be accounted for in modeling the transport of the beam through the accelerator. The purpose of the project is to model the transport of a low energy, specifically between 8 and $100 \mathrm{MeV}$, electron beam through an accelerator, accounting for space charge effects when necessary, to accurately measure the emittance of the beam. 


\section{MATERIALS AND METHODS}

One can analytically transport the beam through solving the differential equations of motion above. However, it is more convenient, and often as effective, to transport the beam through a matrix formalism similar to that of linear optics used in the transport of optical beams. A transfer, or transformation, matrix represents the linear component of the force of a beam transport element, whether it be a quadrupole, bending magnet, or drift space, which is a region between magnets in which no external electromagnetic forces act on the beam. The transport of particle coordinates through that element of the accelerator is given by the product of the coordinate vector, $\left(x, x^{\prime}, y, y^{\prime}, z, z^{\prime}\right)$, and the corresponding transfer matrix of the accelerator element, as below:

$$
\left(\begin{array}{c}
x \\
x^{\prime} \\
y \\
y^{\prime} \\
z \\
z^{\prime}
\end{array}\right)=\left(\begin{array}{cccccc}
C_{x}(s) & S_{x}(s) & 0 & 0 & 0 & 0 \\
C_{x}^{\prime}(s) & S_{x}^{\prime}(s) & 0 & 0 & 0 & 0 \\
0 & 0 & C_{y}(s) & S_{y}(s) & 0 & 0 \\
0 & 0 & C_{y}^{\prime}(s) & S_{y}^{\prime}(s) & 0 & 0 \\
0 & 0 & 0 & 0 & C_{z}(s) & S_{z}(s) \\
0 & 0 & 0 & 0 & C_{z}^{\prime}(s) & S_{z}^{\prime}(s)
\end{array}\right)\left(\begin{array}{c}
x_{0} \\
x_{0}^{\prime} \\
y_{0} \\
y_{0}^{\prime} \\
z_{0} \\
z_{0}^{\prime}
\end{array}\right)
$$

where $\left(x_{0}, x_{0}^{\prime}, y_{0}, y_{0}^{\prime}, z_{0}, z_{0}^{\prime}\right)$, is the initial coordinate vector, $\left(x, x^{\prime}, y, y^{\prime}, z, z^{\prime}\right)$, is the coordinate vector after transport, $C_{\alpha}$ and $S_{\alpha}$ are the solutions of the differential equations of motion for a given transport element, and $C_{\alpha}^{\prime}$ and $S_{\alpha}^{\prime}$ are their derivatives with respect to $s$ [2]. It is also possible to calculate the transport of the second order moments of the beam through the same transfer matrices; however, instead of directly transporting the moments as we do the coordinates above, we create a correlation matrix with indices consisting of the second 
order moments of the coordinate vector. We define the correlation matrix as:

$$
\sigma=\left(\begin{array}{cccccc}
<x^{2}> & <x x^{\prime}> & <x y> & <x y^{\prime}> & <x z> & <x z^{\prime}> \\
<x x^{\prime}> & <x^{\prime 2} & <x^{\prime} y> & <x^{\prime} y^{\prime}> & <x^{\prime} z & <x^{\prime} z^{\prime}> \\
<x y> & <x^{\prime} y> & <y^{2}> & <y y^{\prime}> & <y z> & <y z^{\prime}> \\
<x y^{\prime}> & <x^{\prime} y^{\prime}> & <y y^{\prime}> & <y^{\prime 2}> & <y^{\prime} z & <y^{\prime} z^{\prime}> \\
<x z> & <x^{\prime} z & <y z> & <y^{\prime} z> & <z^{2}> & <z z^{\prime}> \\
\left.<x z^{\prime}\right\rangle & <x^{\prime} z^{\prime}> & <y z^{\prime}> & <y^{\prime} z^{\prime}> & <z z^{\prime}> & <z^{\prime 2}>
\end{array}\right)
$$

[3], and we transport the second order moments through multiplying the transfer matrix by the product of the correlation matrix with the transpose of the transfer matrix, as below:

$$
\sigma=M \sigma M^{T},
$$

[1], where $\mathrm{M}$ is the transfer matrix.

In our case, we focus primarily on the use of quadrupoles, rather than bending magnets, as transfer components. Quadrupole electromagnets, which are focusing in one plane and defocusing in the other, can be placed in an alternating focusing and defocusing pattern along the beam line to provide the appropriate restoring forces necessary to confine the charged particle beams in the transverse plane. We therefore model the beam line as a system of quadrupoles and drift spaces, applying the action of each element to the beam as it travels through the accelerator.

We develop a MATLAB code to model the transport and measure the emittance of low energy electron beams, specifically less than $100 \mathrm{MeV}$, in the accelerator. We do so through the use of transfer matrices and the performance of quadrupole scans to transport both the particles of the beam individually and the beams statistics as a whole, from which we calculate the emittance. We discretize the beamline into separate elements, including the 
quadrupole magnets and drift spaces, the actions of which we represent with different transfer matrices. In the case of a quadrupole that is focusing in the $x$-plane and defocusing in the $y$-plane, the element is represented by the following $6 \times 6$ matrix:

$$
M_{\text {quad }}=\left(\begin{array}{cccccc}
\cos (k l) & \frac{1}{k} \cos (k l) & 0 & 0 & 0 & 0 \\
-k \sin (k l) & \cos (k l) & 0 & 0 & 0 & 0 \\
0 & 0 & \cosh (k l) & \frac{1}{k} \sinh (k l) & 0 & 0 \\
0 & 0 & k \sinh (k l) & \cosh (k l) & 0 & 0 \\
0 & 0 & 0 & 0 & 1 & l \\
0 & 0 & 0 & 0 & 0 & 1
\end{array}\right)
$$

where $k=\sqrt{|K|}$, with $K$ being the focusing strength of the quadrupole magnet, and $l$ is the length of the quadrupole [1]. We represent a drift space through the following transfer matrix:

$$
M_{\text {drift }}=\left(\begin{array}{cccccc}
1 & L & 0 & 0 & 0 & 0 \\
0 & 1 & 0 & 0 & 0 & 0 \\
0 & 0 & 1 & L & 0 & 0 \\
0 & 0 & 0 & 1 & 0 & 0 \\
0 & 0 & 0 & 0 & 1 & L \\
0 & 0 & 0 & 0 & 0 & 1
\end{array}\right)
$$

where $L$ is the length of the drift space [1]. We systematically move along the beamline, separately multiplying the particle coordinate vector by the corresponding transfer matrix that the beam is currently moving through in order to computationally transport the coordinates of the beam. Simultaneously, we transport the statistics of the beam through the same approach, comparing the transported moments with the second order moments of the transported coordinates to confirm their consistency. We model the action of the entire accelerator on the beam through the matrix product of each successive beamline element's 
transfer matrix. However, in cases of low energy or high charge, space charge effects on the beam become non-negligible and must be accounted for in the beam transport in order to accurately calculate the beam emittance. We account for space charge effects by applying a kick to the beam at regular, short intervals and represent these effects through a matrix similar to the transfer matrices above:

$$
M_{S C}=\left(\begin{array}{cccccc}
1 & 0 & 0 & 0 & 0 & 0 \\
\frac{\Delta s}{f_{s c, x}} & 1 & 0 & 0 & 0 & 0 \\
0 & 0 & 1 & 0 & 0 & 0 \\
0 & 0 & \frac{\Delta s}{f_{s c, y}} & 1 & 0 & 0 \\
0 & 0 & 0 & 0 & 1 & 0 \\
0 & 0 & 0 & 0 & \frac{\Delta s}{f_{s c, z}} & 1
\end{array}\right)
$$

where $\Delta s$ is the short interval over which the space charge effects are applied, and $f_{s c}$ is the thin lens focal length due to space charge, defined as:

$$
\begin{aligned}
& \frac{1}{f_{s c, x}}=\frac{K}{2}\left[\frac{1}{5} \frac{1}{\left.<x^{2}\right\rangle}\right]^{3 / 2} R_{D}\left[\frac{\left.<y^{2}\right\rangle}{<x^{2}}, \frac{\left.<z^{2}\right\rangle}{\left.<x^{2}\right\rangle}, 1\right] \\
& \frac{1}{f_{s c, y}}=\frac{K}{2}\left[\frac{1}{5} \frac{1}{<y^{2}>}\right]^{3 / 2} R_{D}\left[\frac{\left.<z^{2}\right\rangle}{<y^{2}}, \frac{\left.<x^{2}\right\rangle}{\left.<y^{2}\right\rangle}, 1\right] \\
& \frac{1}{f_{s c, z}}=\frac{K}{2}\left[\frac{1}{5} \frac{1}{\left.<z^{2}\right\rangle}\right]^{3 / 2} R_{D}\left[\frac{\left.<x^{2}\right\rangle}{<z^{2}}, \frac{\left.<y^{2}\right\rangle}{\left.<z^{2}\right\rangle}, 1\right]
\end{aligned}
$$

where $\mathrm{K}$ is the beam perveance, defined above, and $R_{D}$ is the Carlton elliptic integral of the second kind, defined as

$$
R_{D}(x, y, z)=\frac{3}{2} \int_{0}^{\infty} \frac{d t}{(t+x)^{1 / 2}(t+y)^{1 / 2}(t+z)^{3 / 2}},
$$

[1]. We thus account for space charge effects by transporting the beam over small subsections 
of the beamline through the transfer matrix approach presented above and then applying the space charge effect matrix after each subsection. In other words, we perform the matrix multiplication described above to yield the transported second order moments, which we use, along with the small longitudinal displacement over which we calculated the transport, in the calculation of the space charge matrix. We then transport the beam, either the coordinates or the second order moments directly, through the space charge matrix using the same formalism as without space charge. We mathematically express this method below:

$$
\begin{gathered}
U=M \times U_{0}, \\
U_{S C}=S C \times U=S C \times M \times U_{0},
\end{gathered}
$$

where $U_{0}$ is the initial coordinate vector, $M$ is the transfer matrix, $U$ is the coordinate vector after transport through the transfer element, $S C$ is the space charge effect matrix, and $U_{S C}$ is the coordinate vector after application of space charge effects, and

$$
\begin{gathered}
\sigma=M \times \sigma_{0} \times M^{T}, \\
\sigma_{S C}=S C \times \sigma \times S C^{T}=S C \times M \times \sigma_{0} \times M^{T} \times S C^{T},
\end{gathered}
$$

where $\sigma$ is the correlation matrix. This process of repeatedly transporting the beam is equivalent to representing the action of the entire beam as a single transfer matrix, formed from the product of each successive transfer matrix, as expressed below:

$$
M=M_{s c, n} M_{n} M_{s c, n-1} M_{n-1} \cdots M_{s c, 2} M_{2} M_{s c, 1} M_{1}
$$

From this application of space charge effects, we obtain the true transport of coordinates and moments and thus the correct emmitance of the beam under space charge effects. 
The hope of this project is to develop a code that enables us to reconstitute the initial properties of an actual beam, from which we can then calculate the emittance. Because the divergence of the beam is not easily accessible, we measure the xrms value at a desired point on the beamline. From the method used in individual particle transport, we know that

$$
\left(\begin{array}{c}
x \\
x^{\prime}
\end{array}\right)=M\left(\begin{array}{l}
x_{0} \\
x_{0}^{\prime}
\end{array}\right)
$$

where

$$
M=\left(\begin{array}{ll}
m_{11} & m_{12} \\
m_{21} & m_{22}
\end{array}\right)
$$

is the $x$-related portion of the matrix formed from the product of consecutive beam transport matrices. From the above equation, we see that

$$
\begin{gathered}
x=m_{11} x_{0}+m_{12} x_{0}^{\prime}, \\
x^{2}=m_{11}{ }^{2} x_{0}{ }^{2}+2 m_{11} m_{12} x_{0} x_{0}^{\prime}+m_{12}{ }^{2}{x_{0}}^{2},
\end{gathered}
$$

which we can express as

$$
<x^{2}>=a<x_{0}{ }^{2}>+b<x_{0} x_{0}^{\prime}>+c<x_{0}{ }^{2}>.
$$

We know the coefficients, $a, b$, and $c$, from the indices of $M$, and we strive to determine the initial second order moments of the beam. Thus, we have three unknowns but only one equation. In order to solve for the unknowns, we introduce more equations by varying the beam elements and measuring the corresponding beam sizes. In our case, we varied only the focusing strength of one quadrupole of the system to calculate the xrms values as a function of varying focusing strength. Using MATLAB's lsqcurvefit function, we least square fitted 
the coefficients of the above equation to the squares of the measured xrms values, from which we determined the initial properties and calculated the emittance of the beam. If we exclude the space charge effects matrices from the calculation of $\mathrm{M}$, we should expect to see a difference between the corresponding emittance and the emittance calculated from an M that includes space charge, with the emittance including space charge being the correct value. We also benchmark our code through comparison with simulations in ASTRA [4], a macroparticle based space charge code.

\section{RESULTS}

Through running our code over drift spaces and quadrupoles, we obtained the same results from individual particle transport as from second order moment transport, thus confirming the consistency between the two methods. Both methods yielded the same second order moments through transport along the beam, and therefore the same emittances, enabling us to continue with the faster method of direct second order moment transport. We ran our code over a system of four quadrupoles with length $0.2062 \mathrm{~m}$ and various focusing strengths placed at 2.003, 2.410, 3.216, and 3.6122 meters along a 5.5 meter beamline, comparing our results with those from ASTRA simulations. In the case of a quadrupole system with focusing strengths of $-3.416,2.3,5,801$, and -7 respectively, we found small differences between our distributions transported without regard to space charge and those created in ASTRA simulations (Fig. 1), confirming the necessity of including space charge effects in the transport of a charged particle beam.

Then, using a separate quadrupole system, with quadrupoles of strength $-9.059,-7.865$, and -1.342 for the first, third, and fourth quadrupoles, respectively, we varied the focusing strength of the second quadrupole. We transported the beam at various charges through the system, using seven different focusing strengths, between 9.5 and 11.5, of the second 
quadrupole and calculated the beam envelopes at 4.3 meters for each focusing strength. ASTRA simulations of the xrms values as a function of focusing strength for the system described above are shown in Fig. 2. We fit the curves of this ASTRA simulation using the transfer matrix, $M$, of the beam that excludes space charge effects, thus calculating different normalized emittances for beams of different charges, as we expected (Fig. 3). We then compared this simulated data with the results from our transport code, run over the same setup [Fig. 4]. Using the beam sizes calculated from our transport, we performed the least square fit using both the transfer matrix produced from the product of all beam transfer matrices, including space charge, and the transfer matrix produced without space charge. Similar to the ASTRA data and expected results, we saw a constant emittance from charge to charge when including space charge effects in the matrix, and we saw a changing emittance from charge to charge when excluding space charge effects, which we show in Fig. 5.

We also transported the beam over a single drift space of one meter to better compare our results with ASTRA, slicing the beam along the z-coordinate and transporting each slice individually for various beam energies and charges. We introduced a form factor to the charge of the beam, which we also later introduced in our quadrupole scans, in order to fit the xrms values of the beam to the ASTRA data. In the case of the $100 \mathrm{MeV}$ beam, we were able to closely fit the xrms values to the ASTRA data by applying a factor of .045 to the charge of each slice of the beam. We sliced the beam because in lower energy beams, the spread of the energy is large, meaning the linear approximation applied in calculating the space charge may no longer be valid. We expected the slices to be consistent, in that we would need to apply the same factor to each slice, which was true in the case of the 100 $\mathrm{MeV}$ beam. While each slice and each charge did not exactly fit the ASTRA data for the $100 \mathrm{MeV}$ beam, our data came very close using the .045 form factor, as seen in Fig. 6. We then modeled this drift in a single slice, the results of which are shown in Fig. 7, finding that the necessary form factor was the previously mentioned factor multiplied by the number of 
slices, which in our case was ten. Thus, in modeling the beam without slicing, such as in our quadrupole scans, we used a form factor of .0045.

We also studied an $8 \mathrm{MeV}$ beam, for which our results were much farther off from the ASTRA simulations. The necessary factor used to fit the data was much greater, at 0.45 , and the data still did not fit the expected results well, as seen in Fig. 8.

\section{DISCUSSION AND CONCLUSIONS}

Our initial results indicate a problem in our transport code that is as of yet unclear. Our transported data for the second quadrupole variation does not completely correspond to the ASTRA data for beam charges of 0,250 , and $500 \mathrm{pC}$. Although we were able to demonstrate the necessity of the inclusion of space charge effects in the calculation of the transfer matrix and thus in the reconstitution of the beam emittance by obtaining a constant emittance with respect to changing charge, our calculated emittance differs slightly from that found in the no space charge case of the ASTRA simulation. This is most likely due to that our transported beam does not correspond exactly to the ASTRA simulated data. As is clear from Fig. 2 and Fig. 4, the beam sizes calculated in our transport code are somewhat different from those calculated in ASTRA, even including the form factor derived from the results of our slicing and drift runs. The form factor was introduced because the distribution of the beam after acceleration is not exactly Gaussian, instead having projections closer to parabolic distributions. Thus, we apply the form factor to the charge in order to account for the different beam volume in the calculation of the chare density of the beam, which we use in calculating the space charge effects. It is also important to note that our our calculation of the space charge includes an approximation of the $R_{D}$ elliptic integral, as follows:

$$
R_{D}\left(Z^{2}, Y^{2}, X^{2}\right)=\frac{3}{X Z} \frac{1}{X+Y}\left(1-\xi\left(\frac{\gamma Z}{\sqrt{X Y}}\right)\right)
$$




$$
\begin{gathered}
R_{D}\left(Z^{2}, X^{2}, Y^{2}\right)=\frac{3}{Y Z} \frac{1}{X+Y}\left(1-\xi\left(\frac{\gamma Z}{\sqrt{X Y}}\right)\right), \\
R_{D}\left(X^{2}, Y^{2}, Z^{2}\right)=\frac{3}{X Y Z} \xi\left(\frac{\gamma Z}{\sqrt{X Y}}\right)
\end{gathered}
$$

where $X^{2}=5<x^{2}>, Y^{2}=5<y^{2}>, Z^{2}=5<z^{2}>$, and $\xi(s)$ is defined below:

$$
\begin{gathered}
\xi(s)=\frac{1}{1-s^{2}}\left(1-\frac{s}{\sqrt{1-s^{2}}} \cos ^{-1}(s), s<1\right. \\
\xi(s)=\frac{1}{1-s^{2}}\left(1-\frac{s}{\sqrt{s^{2}-1}} \cosh ^{-1}(s), s>1\right.
\end{gathered}
$$

The gamma factor in the $\xi(s)$ approximation was added in an attempt to better fit our data to ASTRA. This gamma factor corresponds to the Lorentz boost, which appears throughout our equations. We should not have had to introduce this factor here, however, which suggests we may be missing a gamma factor somewhere else in our equations. Once this missing factor is found, our data should fit more correctly. We also encountered problems in our results when running over a simple drift space. We focused on applying a factor to fit the xrms data, but in doing so, our x'rms and zrms data does not match the ASTRA simulated results. Because space charge effects depend on the entire volume of the beam, applying a factor to fit the beam size in $\mathrm{x}$ should have affected all of the rms values in a similar way. That the form factor does not fit the x'rms and zrms values to the ASTRA data suggests another aspect of our code is negatively affecting our data. Similarly, our results from the $8 \mathrm{MeV}$ beam are significantly different from ASTRA data. As of yet, more benchmarking with the $8 \mathrm{MeV}$ code is required to understand the problem. We plan to continue with our code, benchmarking it with ASTRA to determine where the problem lies. We can also perform a convergence study to determine the best number of steps to use in calculating our space charge, which may also affect our results. Upon fixing our code to correctly transport the beam, we will be able to calculate the correct emittances under space charge effects. 


\section{ACKNOWLEDGEMENTS}

I would like to thank my mentor, Cecile Limborg-Deprey, for volunteering her time and effort this summer. I am extremely grateful for her kindness and help throughout the past weeks and for providing me with such an interesting and exciting project to work on. I also thank the Department of Energy and SLAC for sponsoring my research through the SULI program. I especially thank my fellow SULI students, who made this summer unforgettable.

\section{REFERENCES}

[1] C. K. Allen and N. D. Pattengale, "Theory and technique of beam envelope simulation: Simulation of bunched particle beams with ellipsoidal symmetry and linear space charge forces," Los Alamos National Laboratory, Los Alamos, New Mexico, Tech. Rep., August 2001.

[2] H. Wiedemann, Particle Accelerator Physics: Basic Principles and Linear Beam Dynamics, 1st ed. Berlin, Heidelberg: Springer-Verlag, 1993, ch. 4-5, pp. 75-158.

[3] C. K. Allen, "Bunched-beam rms envelope simulation with space charge," January 2005, los Alamos National Laboratory Seminar Presentation.

[4] ASTRA A Space Charge Tracking Algorithm User's Manual, July 2008. 


\section{FIGURES}
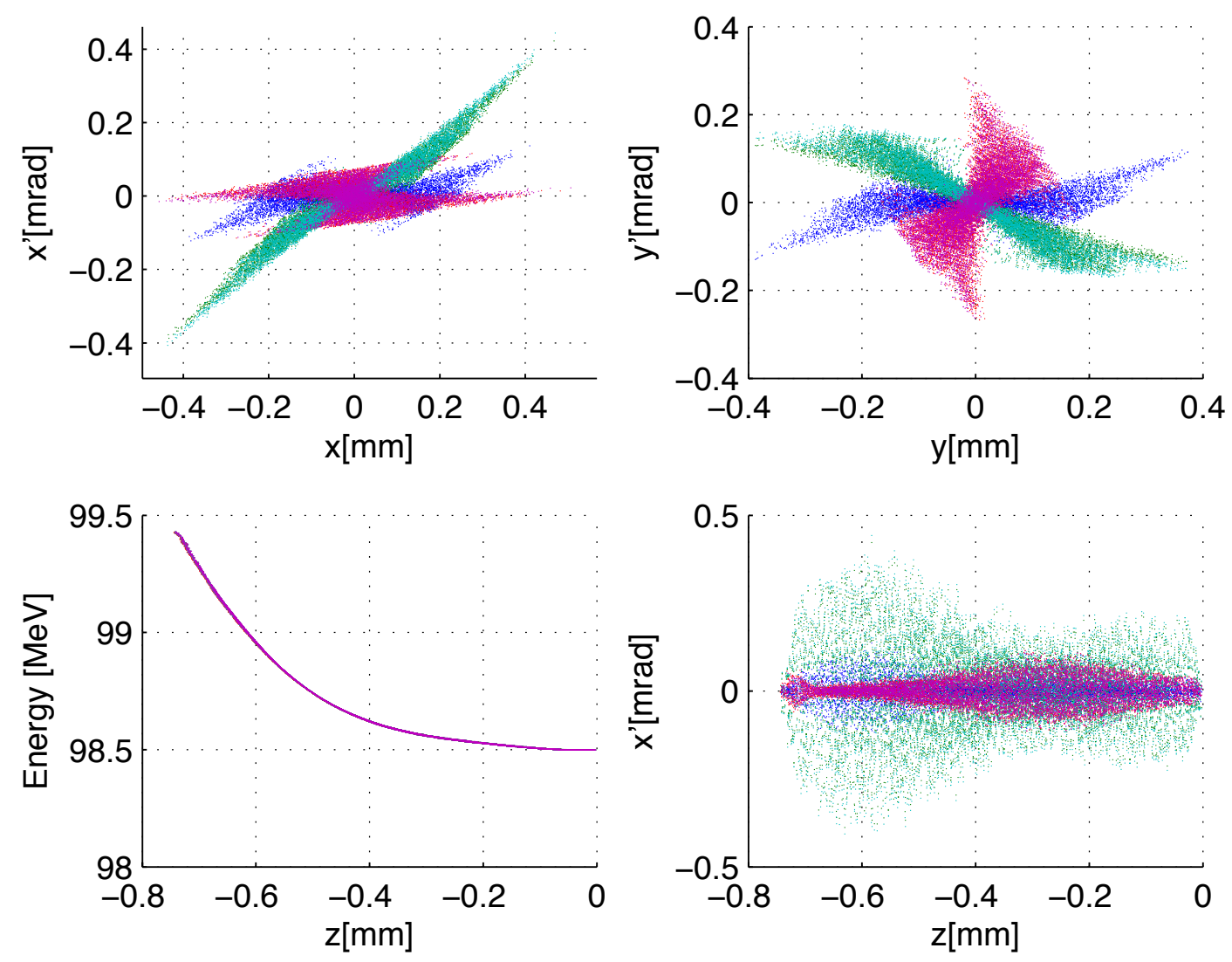

Figure 1: Overlay of transported distributions on ASTRA simulations at $z=1.9 \mathrm{~m}$ (blue), $z=2.1062 \mathrm{~m}$ (green, cyan), or immediately following the first quadrupole, and $z=5.5 \mathrm{~m}$ (red, magenta), or the end of the beamline, for $100 \mathrm{MeV}$ beam. Transported distribution does not take space charge into account. Slight differences between ASTRA and modeled distributions confirm effects of space charge on transport of beam. 


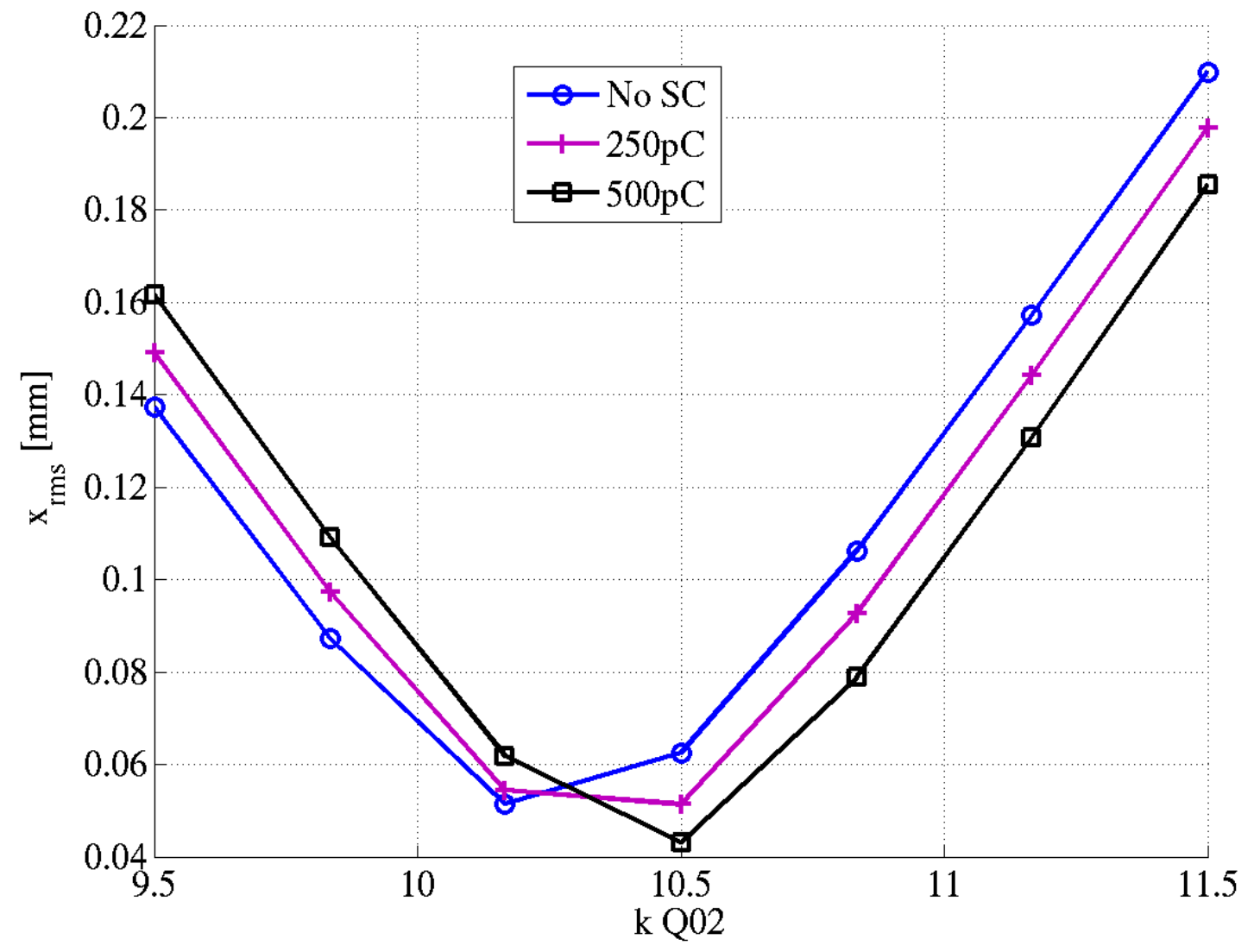

Figure 2: Beam envelope in $\mathrm{x}$ (xrms) of 0,250 , and $500 \mathrm{pC}$ charged beam from ASTRA simulations as function of focusing strength of second quadrupole. Space charge effects clearly influence beam size. 


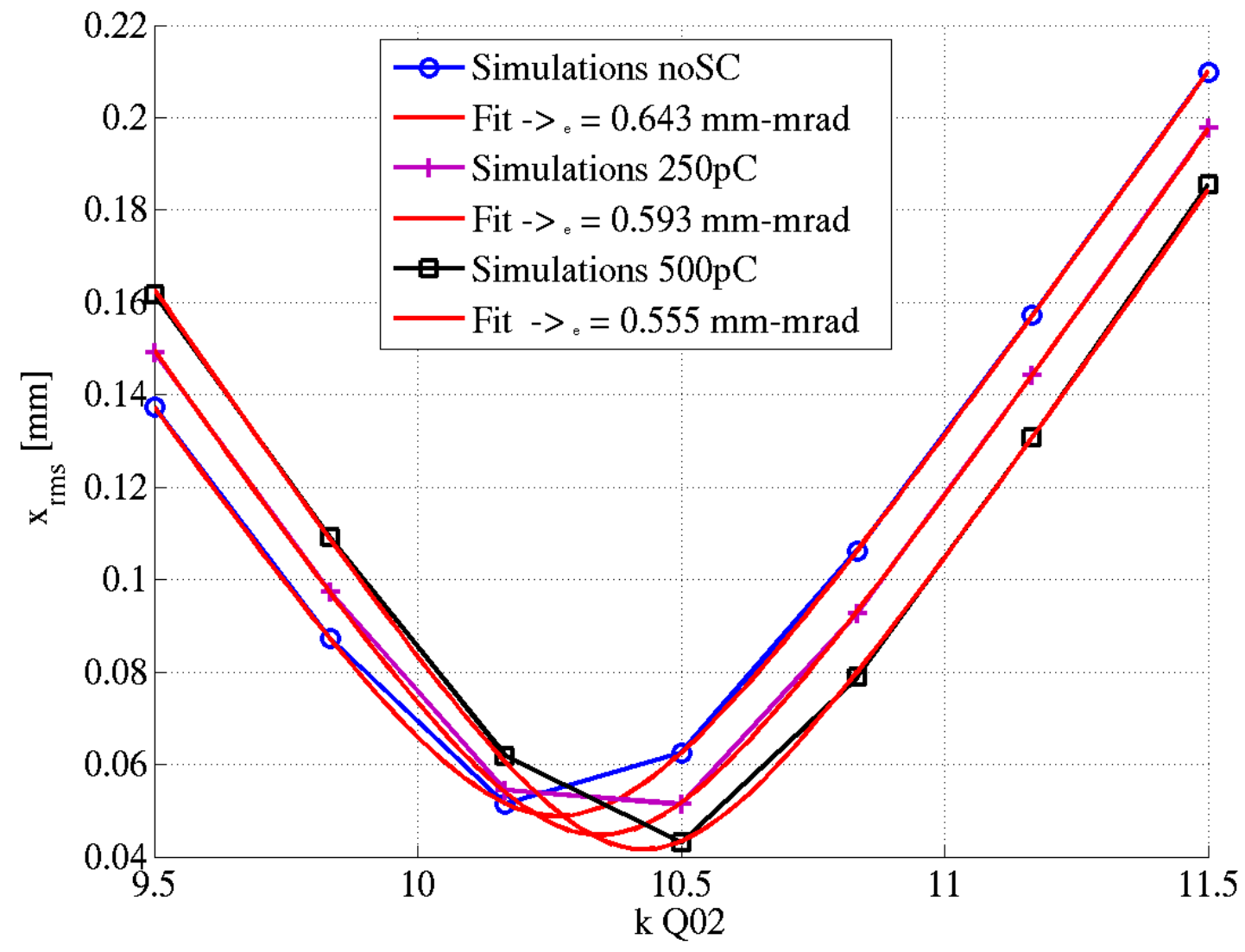

Figure 3: Least square fit of ASTRA simulations of beam envelops in $\mathrm{x}$ (xrms) as function of focusing strength of second quadrupole. Matrix used to least square fit does not include space charge, resulting in changing emittance with increasing beam charge. 


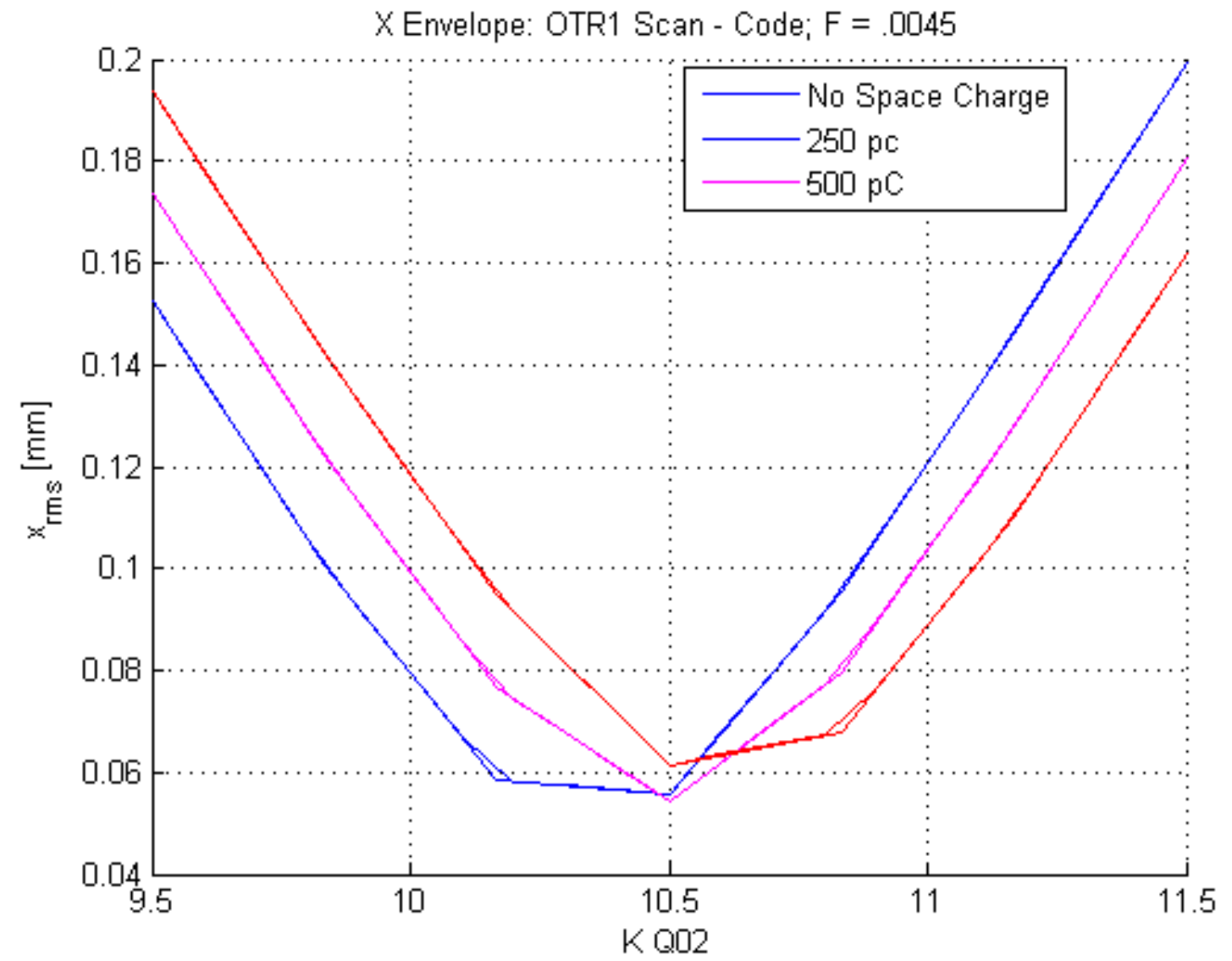

Figure 4: Beam envelope in $\mathrm{x}$ (xrms) of 0, 250, and $500 \mathrm{pC}$ charged beam from MATLAB transport code as function of focusing strength of second quadrupole. Form factor of .0045 introduced to charge to better fit results to ASTRA simulations, although beam sizes still differ from expected values. Regardless, fitting with matrix including space charge still results in constant emittance from charge to charge, although calculated emittance differs slightly from expected value. 


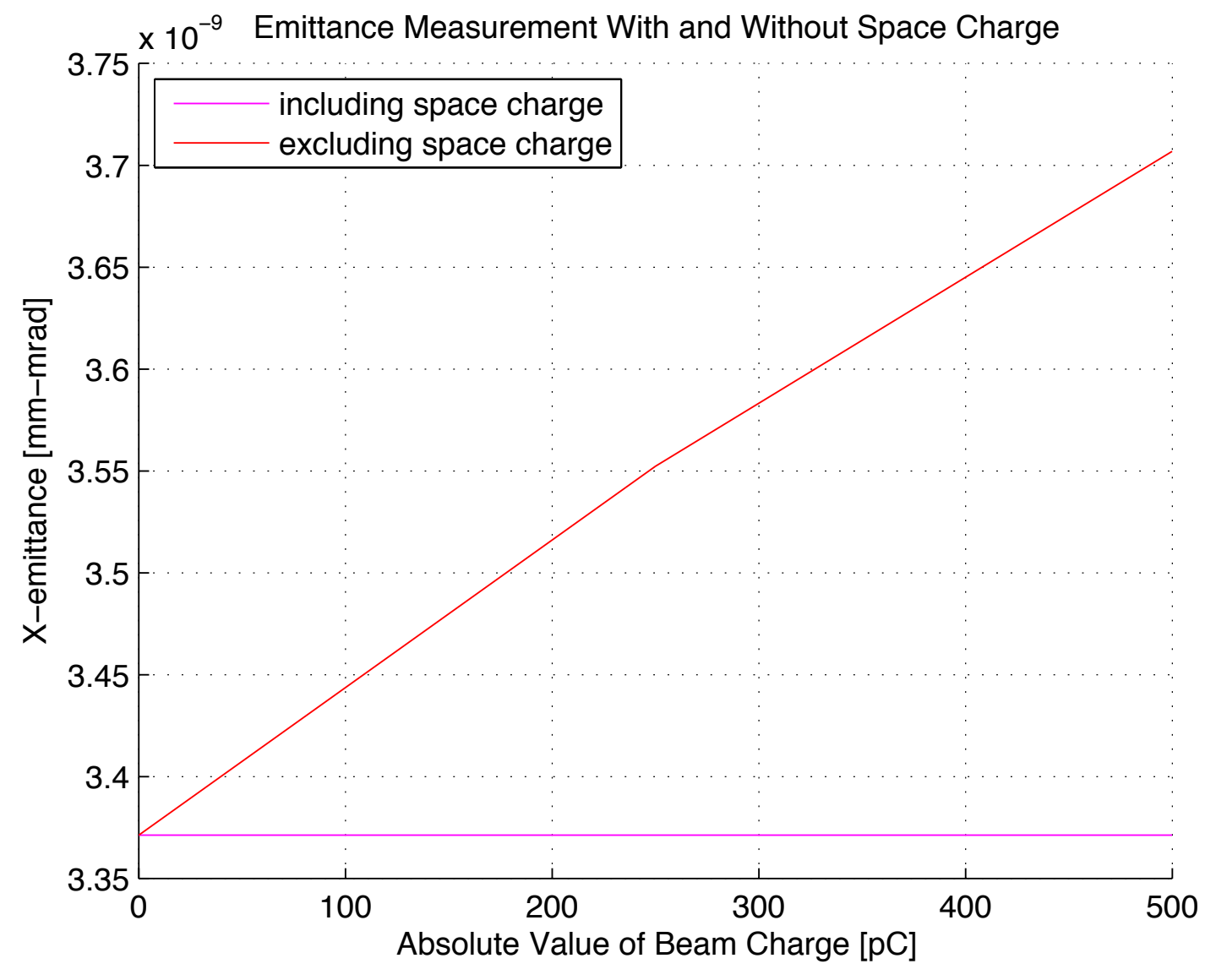

Figure 5: Beam emittance, calculated from least square fit to beam size, as a function of charge. Excluding space charge effects from calculation of transfer matrix, indices of which used as coefficients in least square fit, results in changing emittance with increasing charge. 


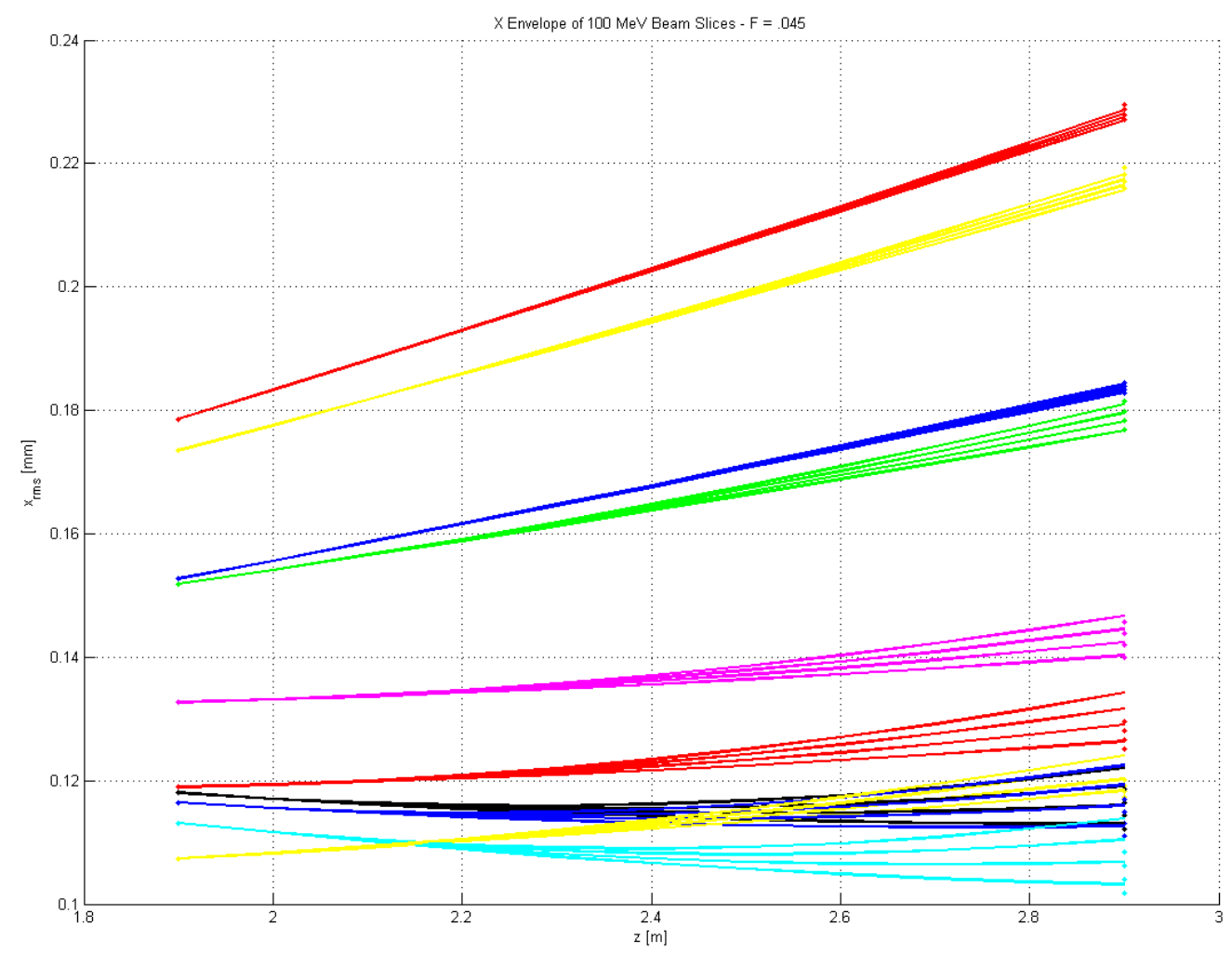

Figure 6: Beam size of 10 z-slices of $100 \mathrm{MeV}$ beam with 250, 500, 750, and $1000 \mathrm{pC}$ charge, respectively, along 1 meter drift. Points represent ASTRA simulated beam sizes at start and end of drift space. Lines represent beam sizes calculated in transport code. Form factor of 0.045 applied to charge for each slice. With increasing charge, difference between transported beam size and ASTRA simulated beam size at end of drift increases. 


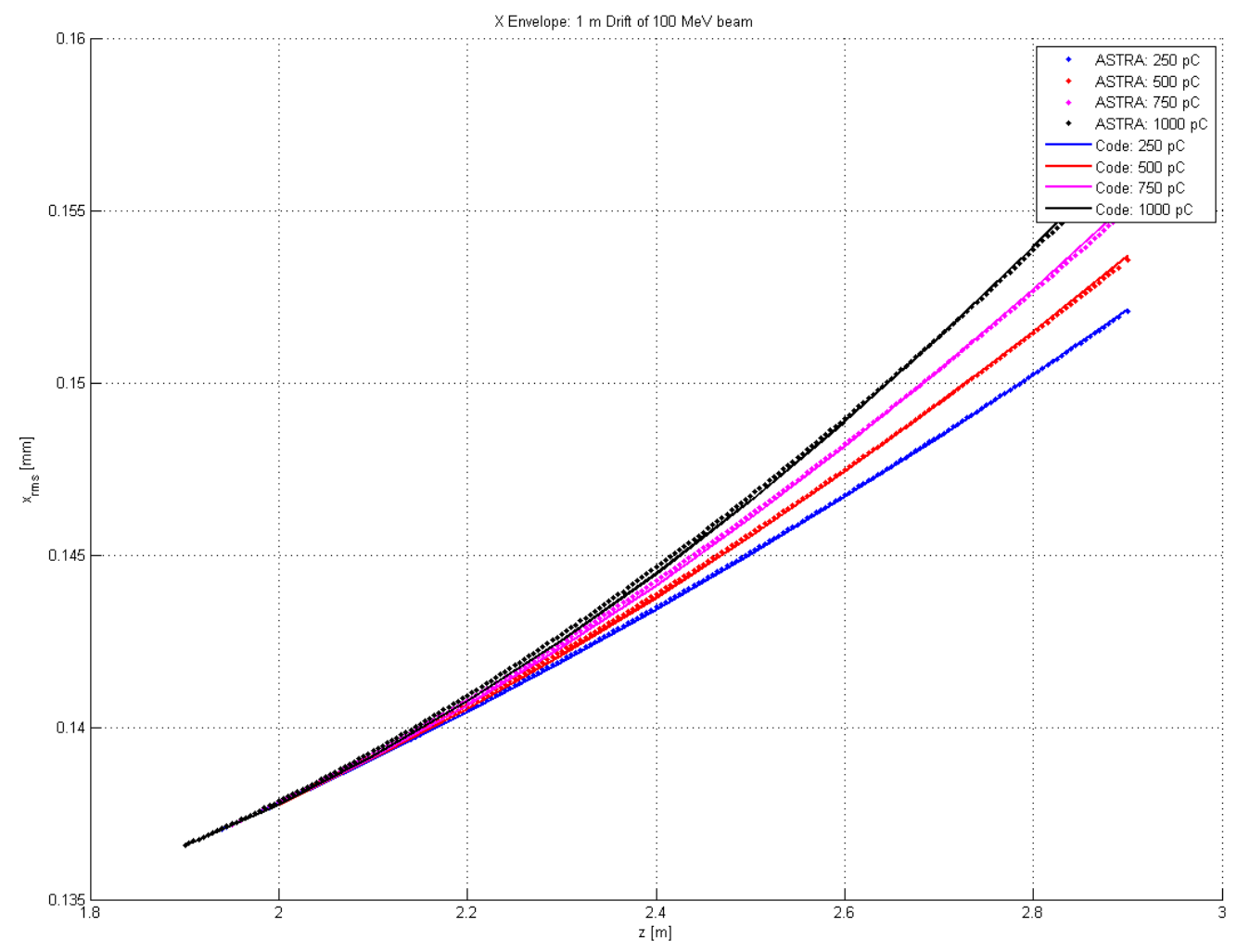

Figure 7: Beam size of $100 \mathrm{MeV}$ beam without slicing and with 250, 500, 750, and $1000 \mathrm{pC}$ charge, respectively, along 1 meter drift. Using 0.0045 form factor on each charge, calculated beam sizes correspond well with ASTRA simulated curves. 


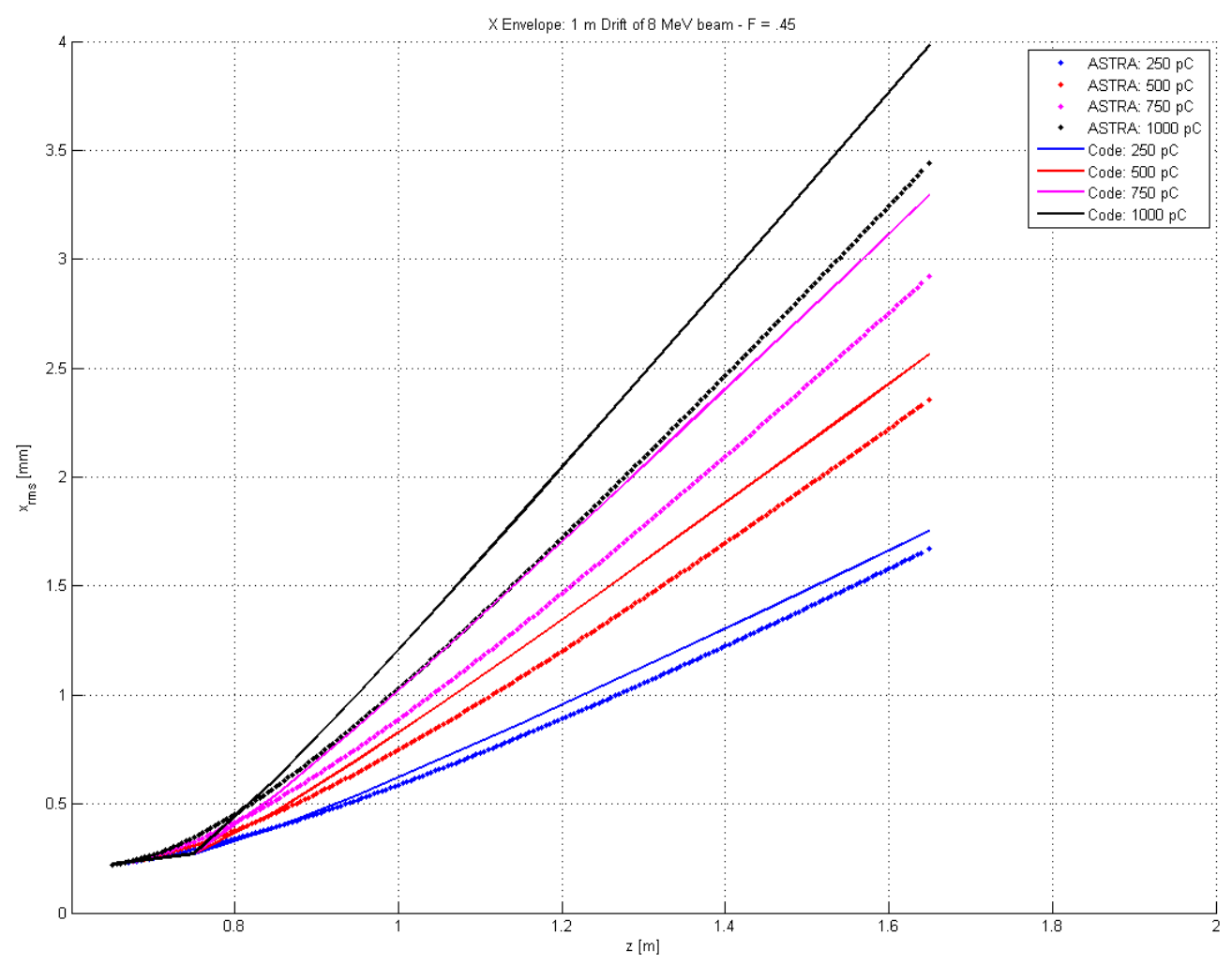

Figure 8: Beam size of $8 \mathrm{MeV}$ beam without slicing and with 250, 500, 750, and $1000 \mathrm{pC}$ charge, respectively, along 1 meter drift. Form factor of 0.45 applied to charge of beam. Clear and significant differences between ASTRA simulated data and data calculated from transport code, with differences in beam size increasing with increasing charge. ASTRA simulates curved growth of beam size along drift, while calculated data models growth of beam size linearly. 